\title{
Fabrication of Superhydrophobic Surface of Alumina with Nanoporous Structure
}

\author{
Wonbae Lee, ${ }^{\dagger, \mathrm{a}}$ Bo Gi Park, ${ }^{\ddagger}$ and Kyu Back Lee ${ }^{\dagger \neq, *,}$ \\ †Department of Biomedical Engineering, College of Health Science, Korea University, Seoul 136-703, Korea \\ ${ }^{*}$ E-mail: kblee@korea.ac.kr \\ ${ }^{\ddagger}$ Department of Interdisciplinary Bio/Micro System Technology, College of Engineering, Korea University, Seoul 136-701, Korea \\ Received January 18, 2010, Accepted May 17, 2010
}

Key Words: Superhydrophobic surface, Nanoimprint, Self-assembled monolayer, Anodized aluminum oxide, Nanoporous pattern

Controlling the wetting property of a surface to be superhydrophobic has been an attractive subject in science and technology ${ }^{1-4}$ Self-cleanable superhydrophobic surfaces promise not only the self-removal of undesired contaminants from the surfaces of high-end products, but also a decrease in the surface energy of the mother molds for the easy departure of a replica pattern in template base lithography.

Anodized aluminum oxide (AAO) is a well-defined nanoporous template to produce ordered pillar patterns ${ }^{5-7}$ or rod-type nanostructures, ${ }^{8-14}$ because the pore size, depth and ordering regime of the resulting pattern can be tuned in the nanometer scale. ${ }^{15-17}$ Fabrication of organized molecular assembly (selfassembled monolayer, SAM) on a micro- or nano-scale structured surface with a low free energy material is an effective approach for obtaining a hydrophobic or a superhydrophobic surface. One technical detail that should be considered in the coating of a SAM on nanoporous surface is the dehydration of nanopores. Because the AAOs are obtained from acidic aqueous solutions and the pore-depth to diameter aspect ratio strongly affects the hysteresis in the permeability and capillary pressure, ${ }^{18}$ dehydration of the water-filled nanopores is crucial for fabricating a well-defined SAM in the pores.

This paper suggests an effective process for fabricating a heptadecafluoro-1,1,2,2-tetrahydrodecyl-trichlorosilane (HDFS) monolayer to reduce the surface energy of porous AAO focusing on its superhydrophobicity and its use as a nanoimprinting template. An ultrapure aluminum plate (Goodfellow, UK) was laser cut into $2 \times 5 \mathrm{~cm}^{2}$ pieces. A well-ordered oxalic AAO (OAAO) mold was fabricated by two-step anodizing in an oxalic acid solution $(0.3 \mathrm{M})$ at $40 \mathrm{~V}$ followed by post-etching for $25 \mathrm{~min}$. The phosphoric AAO (PAAO) mold also was obtained with phosphoric acid (0.1 M) at $193 \mathrm{~V}$. Dehydration of the AAOs was carried out in two different ways. One was 'simple drying' with a gentle stream of $\mathrm{N}_{2}$ gas (OAAO-sd and PAAO-sd), and the other was the more effective ' $\mathrm{MeOH}$-exchange dehydration' (OAAO-md and PAAO-md) with soaking in methanol for more than 1 hour followed by drying in a vacuum desiccator. The plates were then soaked into a $1 \mathrm{mM}$ solution of HSFS (Gelest Inc., Penn.) in $n$-hexane for 1 hour.

The cross-section FE-SEM image of OAAO in Figure 1 (a) shows well-ordered straight nanometer-scale holes (several tens nanometer of the diameter) with a micrometer-scale depth

${ }^{\mathrm{a}}$ These authors contributed equally.
$(3.32 \mu \mathrm{m})$. The fabrication of a self-assembled HDFS monolayer on the surface with the two different dehydrations was investigated using wavelength dispersed spectroscopy (WDS) and contact angle (CA) measurements. The WDS of the OAAOs were obtained by JXA- $8500 \mathrm{~F}$ electron probe micro analyzer (EPMA, Jeol, Japan). The silicon peak, which is due to the HDFS molecular layer, was detected with discrete peaks at both the lower and upper regions of the grown oxide in OAAO-md (b1 and b2 in Figure 1 (b)): The HDFS molecular layer was assembled over the entire area in the pores of OAAO-md, even at the lower parts to a micrometer-scale depth. In contrast, the OAAOsd sample had no meaningful silicon peak from the surface of pores (b3 and b4 in Figure 1 (b)).

The CA measurement revealed more interesting results. The CAs were obtained using an Easydrop goniometer (KRÜSS, Germany) on more than 3 points for each specimen. The PAAOsd had an initial CA of $78.0 \pm 17.7^{\circ}$, which increased dramatically to the superhydrophobic range after post-drying in a vacuum desiccator; $162.0 \pm 1.0^{\circ}$ for 48 hours. This is identical to the final CA of PAAO-md. The CA of OAAO-sd also increased dramatically to $153.0 \pm 1.0^{\circ}$, which is similar to the CA of OAAO-md $\left(152.0 \pm 1.0^{\circ}\right)$. Figure 2 shows the time dependent changes in the CA of OAAO-sd for post-drying. One of the reasonable explanations for these results is that the HDFS molecular layer in the OAAO-sd and PAAO-sd formed only at the uppermost surfaces, but not inside the walls of the pores due to pre-existing water. Therefore, the porous sample was wetted easily by water droplets in the early stages. However, the water

(a)

(b)
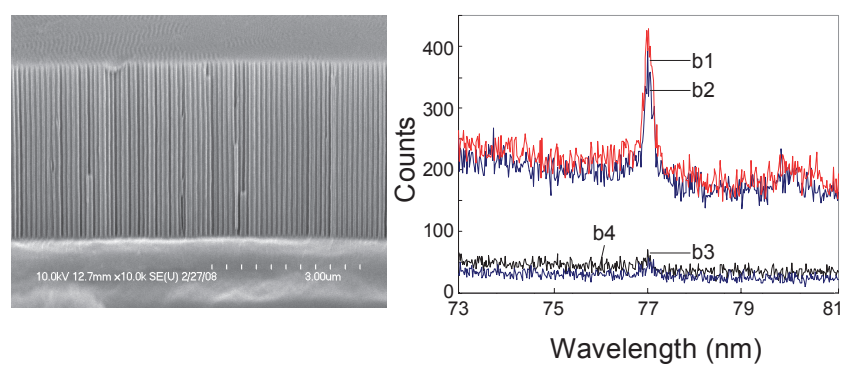

Figure 1. Formation of an HDFS-monolayer on the AAOs: (a) FE-SEM cross section image of the OAAO and (b) the silicon spectra of WDS at the top area of OAAO-md (b1), the bottom area of OAAO-md (b2), the top area of OAAO-sd (b3), and the bottom area of OAAO-sd (b4). 


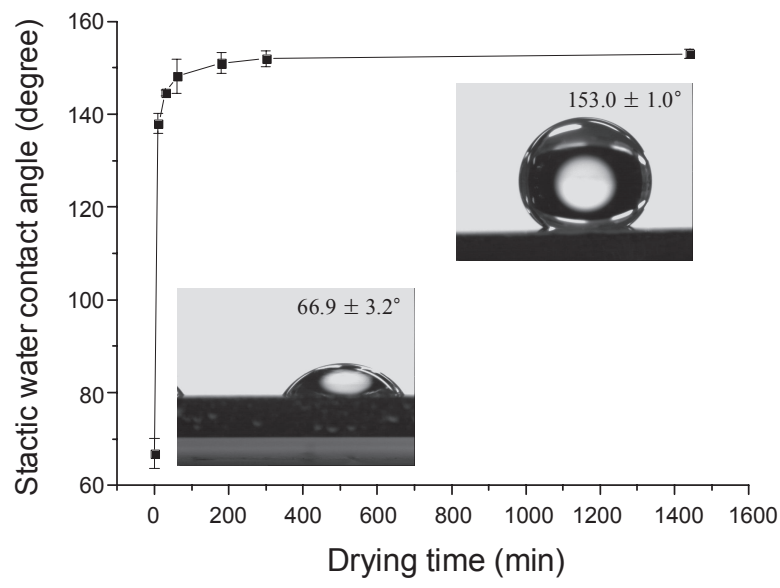

Figure 2. Post-drying time-dependent contact angle of OAAO-sd with a superficial HDFS-coating. The water droplet images on the initially and finally dried OAAO-sd are shown in the box.

droplet was almost completely repelled after the post-drying process, which filled the nanopores with dried air. In addition, the other experimental result can also be understood by the same rationale. A series of OAAO-sd samples with various pore-depths, 420, 1002, 2350 and $3320 \mathrm{~nm}$, had identical final CA values $\left(152.0 \pm 1.0^{\circ}\right)$ after post-drying for 48 hours.

Although the wetting of porous AAO is independent of the HDFS-covering inside the walls, modification of the internal walls with a low free energy material is still important for the successful departure of a replica pattern from the AAO mold. The polystyrene (PS) nanopatterns were imprinted on a ST31 3120 plate (Goodfellow, UK) using a NANOSIS ${ }^{\mathrm{TM}} 610$ (Nano \& Device, Korea) with the AAO molds. Figure 3 shows the FE-SEM images of the nano-imprinted PS patterns from the various OAAO molds after complete post drying in a vacuum desiccator for $48 \mathrm{hrs}$. The AAO molds for the patterns in Figures 3(a) and 3 (b) had the same dimensional features and HDFS SAM covering at the uppermost surface, which had similar CAs of $153.0 \pm 1.0^{\circ}$ and $152.0 \pm 1.0^{\circ}$ after post-drying. However, of the fabrication of HDFS SAM on the inner-pores depends on the dehydration process. The imprinted PS pattern from the simply dried mold (Figure 3 (a)) showed undesirably elongated and rooted up pillar pattern due to the high surface free energy at the inner walls without an HDFS monolayer, whereas the other imprinting pattern from the HDFS-SAM covered OAAO-md mold (Figure 3(b)) showed well-ordered nanopillars.

These results suggest an interesting scientific indication and provide a useful technological criterion for fabricating an organized molecular assembly of a low surface energy material on the porous surface of AAO. The behavior of water droplets on the finally obtained superhydrophobic surfaces of the HDFS monolayer-coated porous AAOs matches well the Cassie state or the intermediate state of the Cassie and Wenzel models because the repellency of surfaces to a water droplet was independent of the HDFS-monolayer coating on the inner-walls of the pores. Technologically, a well fabricated HDFS SAM over the entire surface including the inner walls by effective dehydration is still an important requirement for preparing well-definedimprinting nanopatterns.

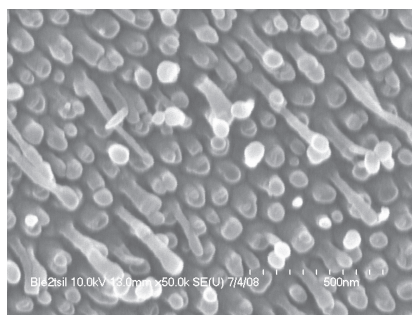

(a)

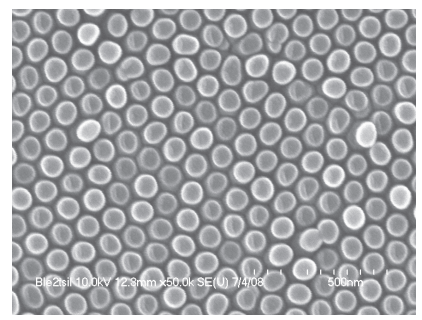

(b)
Figure 3. Imprinted PS patterns from (a) OAAO-sd and (b) OAAOmd molds.

Acknowledgments. This study was supported in part by the National Research Foundation of Korea (NRF) grant funded by the Korea government(MEST) (No. 2010-0014693), by the Seoul R\&BD Program (10920), by the Korea Foundation for International Cooperation of Science \& Technology (KICOS) through a grant provided by the Korean Ministry of Science \& Technology (MOST) in 2009 (No. M60601010002-06E010100210), by the Korea Science and Engineering Foundation through the Pioneer Converging Technology Program (No. M10711160001-08M1116-00110) and by a grant of the Korea Healthcare technology R\&D Project, Ministry of Health, Welfare \& Family Affair, Republic of Korea (A084204).

\section{References}

1. Hong, X.; Gao, X.; Jiang, L. J. Am. Chem. Soc. 2007, 129(6), 1478-1479.

2. Lai, Y.; Gao, X.; Zhuang, H.; Huang, J.; Lin, C.; Jiang, L. Advanced Materials 2009, 21(37), 3799-3803.

3. Blossey, R. Nat. Mater. 2003, 2(5), 301-306.

4. Zhang, H.; Lamb, R. N.; Cookson, D. J. Applied Physics Letters 2007, 91(25), 254106-3.

5. Kim, M.; Kim, K.; Lee, N. Y.; Shin, K.; Kim, Y. S. Chem. Commun. 2007, 22, 2237-2239.

6. Kim, D.; Lee, H.; Lee, J.; Kim, S.; Lee, K.-H.; Moon, W.; Kwon, T. Microsystem Tech. 2007, 13(5), 601-606.

7. Lee, H.; Kim, D.; Kwon, T. Microsystem Tech. 2007, 13(5), 593599.

8. Gao, H.; Mu, C.; Wang, F.; Xu, D.; Wu, K.; Xie, Y.; Liu, S.; Wang, E.; Xu, J.; Yu, D. J. Appl. Phys. 2003, 93(9), 5602-5605.

9. Hu, W.; Gong, D.; Chen, Z.; Yuan, L.; Saito, K.; Grimes, C. A.; Kichambare, P. Appl. Phys. Lett. 2001, 79(19), 3083-3085.

10. Suh, J. S.; Jeong, K. S.; Lee, J. S.; Han, I. Appl. Phys. Lett. 2002, 80(13), 2392-2394.

11. Zhou, Y.; Shen, C.; Li, H. Solid State Ionics 2002, 146(1-2), 81-86.

12. Zhou, Y.; Huang, J.; Shen, C.; Li, H. Mater. Sci. Eng. A 2002, 335 (1-2), 260-267.

13. Zhang, Q.; Chakraborty, A. K.; Lee, W. I. Bull. Korean Chem. Soc. 2009, 30(1), 227-229.

14. Jung, J.-S.; Malkinski, L.; Lim, J.-H.; Yu, M.; O'Connor, C. J.; Lee, H.-O.; Kim, E. M. Bull. Korean Chem. Soc. 2008, 29(4), 758-760.

15. Furneaux, R. C.; Rigby, W. R.; Davidson, A. P. Nature 1989, 337 (6203), 147-149.

16. Patermarakis, G.; Moussoutzanis, K. Corros. Sci. 2001, 43(8), 1433-1464.

17. Chu, S. Z.; Wada, K.; Inoue, S.; Isogai, M.; Katsuta, Y.; Yasumori, A. J. Electrochem. Soc. 2006, 153(9), B384-B391.

18. Jerauld, G. R.; Salter, S. J. Transport Porous Med. 1990, 5(2), 103-151. 